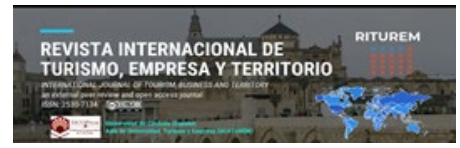

Cita bibliográfica: Fernández, C. y Salinas, E.R. (2020). El Centro de Análisis y Prospectiva del Turismo en Córdoba: un proyecto experimental de investigación y transferencia de conocimiento para el sector turístico. Revista Internacional de Turismo, Empresa y Territorio, 4 (1), 124-134. https://doi.org/10.21071/riturem.v4i1.12863

\title{
El Centro de Análisis y Prospectiva del Turismo en Córdoba: un proyecto experimental de investigación y transferencia de conocimiento para el sector turístico
}

\section{The Center for Tourism Analysis and its Prospectives in Córdoba: an experimental project of research and knowledge transfer for the tourism sector}

\author{
Carmen Fernández Cañero $1^{1 *}$ \\ Enrique Rafael Salinas Cuadrado $2^{2}$
}

\section{Resumen}

El Centro de Análisis y Prospectiva del Turismo (CAPT) ha sido creado a iniciativa del Vicerrectorado de Investigación y Desarrollo Territorial y la Oficina de Transferencia de la Investigación (OTRI) de la Universidad de Córdoba, con la cofinanciación inicial de la Secretaría General de Universidades, Investigación y Tecnología de la Consejería de Economía, Conocimiento, Empresas y Universidad de la Junta de Andalucía, así como con los fondos FEDER de la Unión Europea. El objetivo principal es recopilar, procesar, analizar, interpretar, sistematizar y divulgar información sobre el sector turístico provincial; apoyando la investigación y su gestión científica, así como facilitando la transferencia de conocimientos de los Grupos de Investigación y expertos de la Universidad de Córdoba tanto a los organismos públicos con competencias en turismo como al sector privado empresarial. Para ello este centro tiene la intención de convocar reuniones científicas y técnicas con las instituciones públicas de turismo como con las y asociaciones empresariales privadas del sector, fomentando el debate y el intercambio de experiencias, así como la investigación multidisciplinar que permita diagnosticar la situación actual y la toma de decisiones a los agentes turísticos cordobeses. Finalmente cabe destacar como principal proyecto del CAPT el sistema de información turística de Córdoba (SITCOR) herramienta de análisis y prospección que ofrecerá resultados periódicos de la actividad turística.

Palabras clave: transferencia; investigación; sistema de información turística; herramienta de análisis, Córdoba

\begin{abstract}
:
The Center for Tourism Analysis and its Prospectives (CAPT) has been created at the initiative of the Vice-Chancellor of Research and Territorial Development and the Research Transfer Office (OTRI) of the University of Córdoba, with the initial co-financing of the General Secretariat of Universities, Research and Technology of the Ministry of Economics, Knowledge, Business and University of the Junta de Andalucía, as well as with the EFRD of the European Union. The main objective is to collect, process, analyze, interpret, systematize and disseminate information about the provincial tourism sector; supporting research and its scientific management, as well as facilitating the

\footnotetext{
${ }^{1}$ Técnico Centro de Análisis y Prospectiva del Turismo de la Universidad de Córdoba, España autora 1. E-mail de contacto: z02fecac@uco.es * Autor para la correspondencia

${ }^{2}$ Técnico Centro de Análisis y Prospectiva del Turismo de la Universidad de Córdoba, España autor 2. E-mail de contacto: u22sacue@uco.es
} 
transfer of knowledge from research groups and experts of the University of Córdoba in the study of tourism policy and planning. To this end, scientific and technical meetings will be convened with public institutions with competence in tourism, as well as with private entities promoting debate and exchange of experiences, as well as multidisciplinary research to diagnose the current situation and decisionmaking for tourism agents in Córdoba. Finally, the tourist information system of Córdoba (SITCOR) analysis and prospecting tool that will offer periodic results of the tourist activity will be highlighted as the main project.

Keywords: transfer; research; tourist information system; analysis tool, Córdoba

\section{Introducción}

En el año 2016 se puso en marcha, en el seno de la Universidad de Córdoba (España), un grupo de trabajo interno formado por investigadores profesorado de distintas áreas de conocimiento especializados en la investigación turística. El grupo estaba enfocado al sector turístico con el objeto de aportar conocimiento científico y mejorar el crecimiento sostenible de una actividad económica destacada en la provincia de Córdoba. Es dentro de este grupo de trabajo, donde surgió la idea de la creación de un Centro para el análisis y la prospectiva del turismo, capaz de aportar información actualizada y periódica que complementase y ampliase las acciones tanto del antiguo Observatorio Turístico del ya desaparecido Consorcio de Turismo del Ayuntamiento de Córdoba, como del también ya inexistente Observatorio creado en su día por el Patronato Provincial de Turismo de Córdoba, pero con un plan de trabajo mucho más ambicioso de transferencia de conocimiento al sector turístico en general de Córdoba provincia y subsectores de actividad económica afines o relacionados con el turismo..

Fue en el mes de enero del año 2020 cuando finalmente se ha puesto en marcha el Centro de Análisis y Prospectiva del Turismo (CAPT) de la Universidad de Córdoba a iniciativa del Vicerrectorado de Investigación y Desarrollo Territorial y la Oficina de Transferencia de la Investigación (OTRI), con la cofinanciación inicial de la Secretaría General de Universidades, Investigación y Tecnología de la Consejería de Economía, Conocimiento, Empresas y Universidad de la Junta de Andalucía, así como los fondos FEDER de la Unión Europea, a través de los incentivos para acciones de transferencia de conocimiento entre los agentes del Sistema Andaluz del Conocimiento y el tejido productivo (Plan PAIDI de la Junta de Andalucía).

El objetivo principal de este proyecto es recopilar, procesar, analizar, interpretar, sistematizar y divulgar información sobre el sector turístico de Córdoba y provincia; apoyando la investigación y su gestión científica, así como facilitar la transferencia de conocimientos sobre turismo al mundo empresarial y a las administraciones públicas.

En cuanto a los objetivos específicos se han previsto los siguientes:

- Conocer en profundidad la situación del sector turístico cordobés y andaluz e informar de ella a través de publicaciones periódicas o monográficas para que las instituciones, tanto públicas como privadas, tengan herramientas disponibles para optimizar la gestión turística y difundir propuestas de mejora para el diseño de un desarrollo turístico sostenible y socialmente responsable tanto a nivel provincial como autonómico.

- Promover y convocar reuniones científicas y técnicas con agentes del sector para debatir sobre el turismo y sus múltiples repercusiones tanto a nivel social, como económico y político, favoreciendo así la mejora de la actividad científica relacionada con el turismo e impulsando su transferencia hacia el tejido socioeconómico. 
- Fomentar la investigación multidisciplinar en el ámbito del sector turístico y estimular programas de colaboración interdepartamentales en el seno de la Universidad de Córdoba y entre éstos y las empresas y AAPP en el ámbito de la investigación aplicada sobre turismo.

- Contribuir a la participación activa de los investigadores de la Universidad de Córdoba en proyectos locales, provinciales, regionales, nacionales y trasnacionales en investigación del sector turístico, apoyando la internacionalización de los grupos de investigación en esta materia.

- Diagnosticar las necesidades formativas y carencias que tienen las empresas de turismo y las propias AAPP con competencias en esta materia. Asimismo, diseñar, planificar, desarrollar y promover programas y acciones formativas desde el ámbito universitario específicas para el sector turístico, en posible colaboración con entidades e instituciones locales, provinciales, regionales, etc.

- Fomentar el intercambio de experiencias y/o opiniones sobre las actuales necesidades o carencias con las que se encuentra este sector de cara a su sostenibilidad y responsabilidad social.

- Aportar datos reales y continuados en el tiempo, acerca de las características de la actividad que desempeñan el turismo, con el objeto de tratar de mejorar la competitividad del propio destino turístico (Córdoba y Andalucía) y de las empresas y profesionales que lo integran.

- Diagnosticar la situación actual y potencialidades de género en el turismo, así como estudiar el papel de la mujer en este sector, con especial atención a la brecha salarial y las desigualdades socio-profesionales que afectan a este colectivo.

- Fomentar la investigación y el desarrollo de nuevas "expresiones" de turismo sostenible, como el turismo agroindustrial y el turismo de naturaleza y activo-deportivo (ecoturismo, turismo cinegético, turismo de pesca, deportes de naturaleza y aventura, etc.), potenciales motores de desarrollo para el entorno socioeconómico andaluz y cordobés.

- Facilitar mediante informes de coyuntura turística y otros trabajos de carácter sectorial la información suficiente y de calidad que sirva de base a la toma de decisiones de los diferentes agentes económicos implicados en el sector turístico, al tiempo que permita evaluar el impacto que las políticas públicas e iniciativas de cualquier otra índole que pudiesen tener sobre la mencionada industria.

- Fomentar el debate entre los diferentes agentes económicos que componen el sector (Administración, asociaciones, empresarios, etc.) a través de reuniones periódicas en las que se presentarán y discutirán los resultados obtenidos y las posibles estrategias a desarrollar.

- En definitiva, fomentar las relaciones entre investigadores y empresas vía estudios y articulación de contratos de transferencia e investigación con la intermediación del Centro de Análisis y Prospectiva del Turismo.

\subsection{Estructura organizativa}

El equipo de trabajo del CAPT está formado por profesores e investigadores de la Universidad de Córdoba con amplio conocimiento del sector turístico. En concreto, su estructura es la siguiente: 
- Presidencia del Centro, de carácter representativo (el Vicerrectorado de Investigación y Desarrollo Territorial de la Universidad de Córdoba).

- Director/a ejecutivo, encargado de coordinar las distintas actuaciones que se lleven a cabo desde el CAPT, así como las actividades e iniciativas del profesorado y personal investigador integrados en el Centro. Esta figura corresponde a un profesor/a perteneciente a la plantilla del Personal Docente e Investigador (PDI) de la Universidad de Córdoba, elegido por acuerdo del Consejo de Gobierno de la UCO.

- Comité Asesor, constituido por profesores e investigadores especializados en turismo y representantes de aquellos centros universitarios, Cátedras, Aulas, etc. con importante implicación y responsabilidades en el ámbito de la docencia, la investigación y la transferencia de conocimiento en materia de turismo dentro de la UCO.

- Gabinete técnico-administrativo de apoyo, conformado por titulados universitarios con experiencia y conocimientos en materia de turismo, contratados y/o colaboradores, que se encargarán del soporte administrativo, técnico y de comunicación necesario para el desarrollo de las actividades del Centro de Análisis y Prospectiva del Turismo.

Figura 1. Estructura Organizativa CAPT-SITCOR. Junio 2020

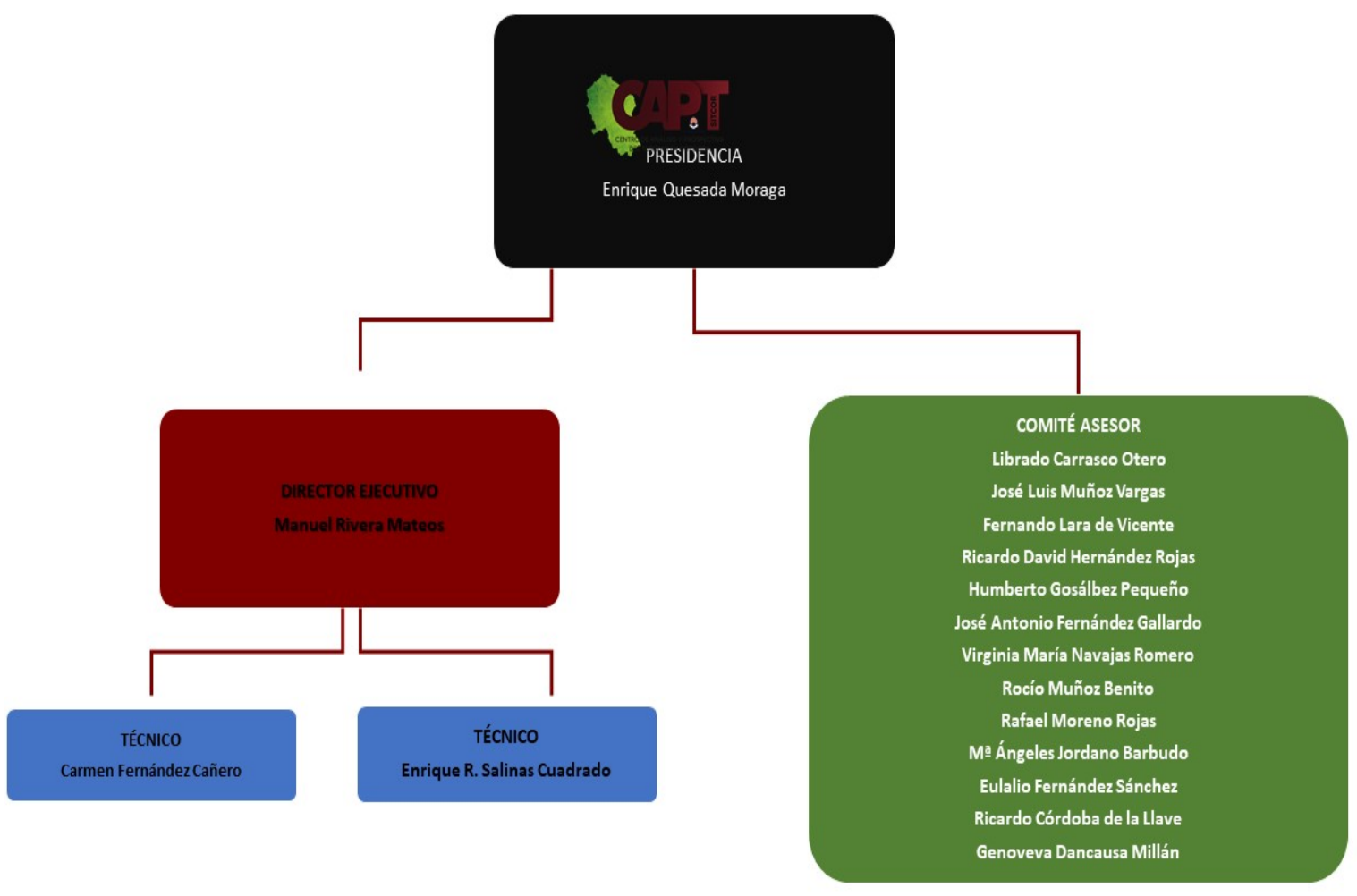

Fuente: Elaboración propia

\subsection{Entidades colaboradoras}

La óptima transferencia de información y conocimiento, la identificación de líneas de investigación prioritarias, así como la consecución de financiación para la consolidación del CAPT hacen necesario el establecimiento de acuerdos marco de colaboración y/o convenios específicos con los agentes turísticos de la provincia de Córdoba. 
El primer convenio marco de colaboración se ha firmado entre la Universidad de Córdoba y el Instituto Municipal de Turismo (IMTUR) del Ayuntamiento de Córdoba siendo formalizado el pasado día 1 de abril del presente año. En dicho convenio se recoge por ambas instituciones "su compromiso de colaboración en los campos científico, técnico y de asesoramiento continuo de interés común en materia de fortalecimiento, competitividad y sostenibilidad del destino turístico". A continuación, se establecerán convenios específicos que aborden proyectos según los objetivos del IMTUR.

\section{EI Sistema de información turística de Córdoba, SITCOR}

El sistema de información turística de Córdoba, SITCOR, es el principal proyecto del Centro Análisis y Prospectiva del Turismo de la Universidad de Córdoba. El desarrollo de esta herramienta de análisis y prospección se contempla para resolver las necesidades de información y conocimiento del sector turístico de Córdoba tanto en el ámbito público como en el ámbito privado.

SITCOR se define como un sistema de recogida de información cuantitativa y cualitativa en la que se emplearán fuentes tanto primarias como secundarias que procederán de estadísticas oficiales, estudios, encuestas e informes de consultoras nacionales, asociaciones y clusters empresariales, así como organizaciones turísticas. Se obtendrá un análisis periódico de la actividad turística enfocado a las necesidades de conocimiento planteadas tanto por las entidades colaboradoras ya sean administraciones públicas o entidades privadas como en las líneas de acción planificadas desde el propio CAPT. Además, se han establecido cuatro áreas básicas de información: análisis de la demanda, estudio de la oferta, impactos socioeconómicos y territoriales y un área de investigación, desarrollo e inteligencia turística.

\section{Figura 2. Áreas básicas de información}

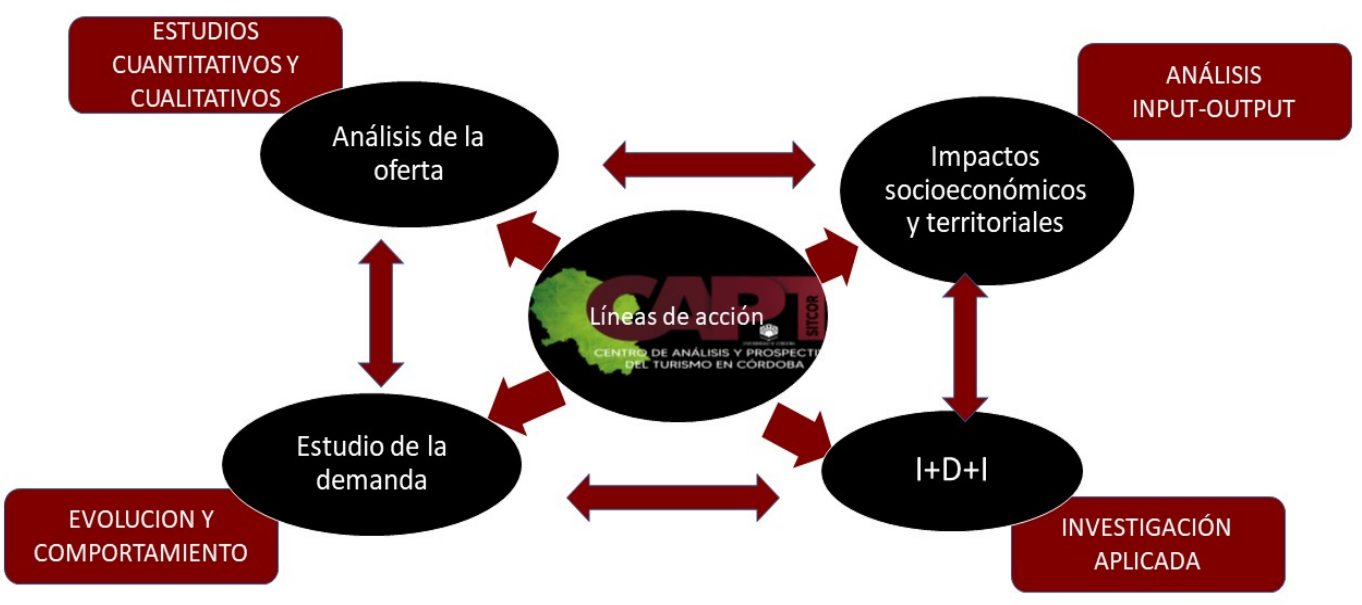

Fuente: Elaboración Propia 


\subsection{Análisis de la demanda y Estudio de la oferta}

El conocimiento del perfil de la demanda turística, así como de la oferta es una necesidad permanente de las entidades provinciales (Diputación Provincial, Mancomunidades de Municipios, Grupos de Desarrollo Rural, Ayuntamientos y ELAs) así como del sector empresarial. Es por ello que SITCOR, se ha planificado como un facilitador de la información en dos vertientes: como herramienta de análisis multidimensional y como un cuadro de mando integral.

\subsubsection{Herramientas de análisis multidimensional}

Se trataría de una herramienta que facilitaría el estudio de la información de los destinos turísticos cordobeses para todos los gestores públicos de ámbito municipal, comarcal o provincial ofreciendo:

- Disponer de información actualizada sobre la oferta y la demanda, sus dinámicas y tendencias.

- Facilitar a las Áreas Municipales y Oficinas de Turismo de la provincia de forma sencilla análisis propios de sus destinos sin tener conocimientos tecnológicos ni estadísticos, a la vez que asesorar a la hora de realizar diagnósticos, diseñar cuestionarios o encuestas sobre la oferta y la demanda o implementar sistemas de información turística local.

- Crear tablas y gráficos dinámicos, con información exhaustiva y atractiva en base a todas las posibilidades de análisis que presenta la información turística y hacer más asequible y comprensible la divulgación de dicha información para públicos amplios y no especializados.

- Alcanzar el máximo nivel de granularidad y detalle de los datos turísticos a nivel provincial y municipal para facilitar la toma de decisiones a empresas y Administraciones Públicas en materia de turismo.

- Realizar análisis comparables entre los datos y estadísticas existentes de los diversos municipios y comarcas de la provincia, regularizando y homogeneizando los instrumentos de obtención de información de las Oficinas y Áreas de Turismo de los Ayuntamientos, Mancomunidades de Municipios y Grupos de desarrollo rural.

\subsubsection{Cuadro de mando integral}

Se obtendrán datos desagregados de la capital y de la provincia considerando las siguientes fuentes:

- Indicadores sobre la oferta de establecimientos y servicios turísticos y la demanda nacional y extranjera.

- Indicadores de diferentes categorías que reflejen el estado de los destinos turísticos provinciales, así como los posibles impactos territoriales de la actividad turística (socioeconómicos y de empleo, socioculturales, ambientales y urbanos, incidencia real del consumo turístico en el destino, etc.).

- Estudios y trabajos de campo específicos sobre la demanda y la oferta (encuestas en destino), de carácter general o por subsectores de actividad (análisis sectoriales) e intentando detectar motivaciones y tendencias de la demanda, mediante un proceso de investigación bien diseñado metodológicamente. 
- Explotaciones específicas y a medida partiendo de las estadísticas oficiales existentes (INE, IECA, etc.), de las bases de datos o sistemas Big Data de las Administraciones turísticas (DataTur, Smart Data Andalucía...) y de las propias Redes Sociales e Internet.

- Metadatos, informes de consultoras o de organizaciones públicas o privadas nacionales o internacionales, así como artículos de revistas científico-académicas, técnicoprofesionales, estudios de investigación universitarios, congresos y jornadas, etc

\subsection{Impactos socioeconómicos y territoriales}

Los estudios e informes realizados contemplarán la incidencia en el territorio respecto al desarrollo económico y la generación de empleo, así como en todos en los ámbitos: ambiental, paisajístico, sociocultural y patrimonial debido al carácter transversal del turismo. Asimismo, se contemplará la problemática de los impactos negativos en el tejido urbano (gentrificación turística, masificación turística e impacto en los alquileres residenciales, consumo e IPC, conflictos con comunidades de vecinos, influencia en el pequeño comercio tradicional, etc.) y en el tejido sociolaboral (precarización laboral, brecha salarial de género, etc.).

\subsection{Investigación, Desarrollo e Innovación Turística}

Esta área de estudio permitirá no solo alcanzar los objetivos de un observatorio turístico tradicional como herramienta de conocimiento turístico continuo, sino que se pretende conformar como un auténtico organismo de "inteligencia turística" que servirá de instrumento de transmisión de conocimiento y de innovación tecnológica en el ámbito de las telecomunicaciones, promoción y comercialización turísticas, mejora de la gestión empresarial, diseño y estructuración de nuevos productos y servicios turísticos, sostenibilidad de empresas y destinos, gestión pública del turismo, ordenación y planificación de los espacios turísticos, minimización de impactos territoriales negativos, etc.

\section{Figura 3. Ámbitos de estudio de Investigación, desarrollo e innovación turística}

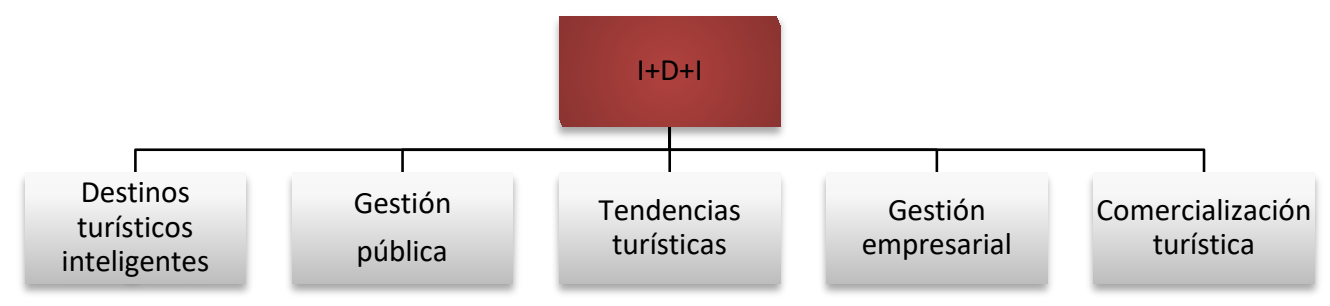

Elaboración propia

Asimismo, se concibe como un espacio colaborativo en el que profesores e investigadores especializados en el ámbito del turismo de la Universidad de Córdoba, el sector público y el sector empresarial turístico de la provincia, consensuen aplicaciones y soluciones adecuadas para mejorar e innovar la oferta de los destinos turísticos intraprovinciales a fin de integrar al visitante con el destino, mejorar la calidad de su estancia y de sus experiencias, propiciar la generación de beneficios socioeconómicos reales para sus comunidades locales. En definitiva, facilitar el desarrollo sostenible de nuestros territorios a través del turismo, 
contribuyendo a mejorar los procesos de planificación estratégica de los destinos y las empresas turísticas de Córdoba y provincia.

Desde el CAPT, se han establecido ocho líneas de acción que serán complementadas con las propuestas que sean consensuadas con los agentes turísticos de la provincia de Córdoba y que se detallan a continuación:

- Asesoramiento de Proyectos de cooperación supramunicipal en materia de redes y clusters turísticos que pretendan configurar o consolidar rutas intraprovinciales e interprovinciales, macroproductos, clubes de producto, etc.

- Asesoramiento en materia de formación y capacitación profesional en turismo tanto para personal de empresas como de Áreas de Turismo y OITs locales y supramunicipales, asociaciones y Centros de Iniciativas Turísticas, etc.

- Diseño y organización de jornadas, seminarios, encuentros y otros eventos a medida de las necesidades del sector turístico provincial.

- Análisis territorial del turismo en la provincia y sus diferentes destinos asesorando en puesta en valor, sostenibilidad ambiental, social y económica en las estrategias de gestión y comercialización de productos y recursos turísticos por comarcas, áreas geográficas, parques naturales, otros espacios protegidos, embalses y pantanos y otras zonas húmedas, Red Natura 2000, espacios seranos, cauces fluviales, etc.

- Calidad de los establecimientos y servicios turísticos, tanto privados y empresariales como públicos y del destino globalmente considerado.

- Impacto turístico de la organización de eventos festivos, culturales, feriales, etc.

- Gestión Pública del Turismo. Asesoramiento a organismos públicos del turismo en materia de gestión pública del turismo de los mismos, establecimiento de indicadores de turismo sostenible y Objetivos de Desarrollo Sostenible (ODS), ejecución de proyectos y captación de ayudas y subvenciones públicas, instrumentación de planes estratégicos municipales o supramunicipales de turismo e implementación de acciones de promoción y colaboración público-privada con la oferta turística local.

- Análisis y prospectiva sobre nuevas tendencias, productos y segmentos turísticos, incidiendo en las potencialidades de la provincia de Córdoba para el desarrollo de los mismos, y especialmente los que se detallan a continuación:

\section{Tabla 1. Segmentos turísticos potenciales}

Turismo colaborativo y economía sumergida: rentabilidad social versus rentabilidad empresarial

Turismo y Patrimonio cultural e histórico-artístico. Turismo y Conjuntos Históricos y centros urbanos de interés

Turismo de naturaleza, ecoturismo y turismo activo en espacios protegidos.

Arqueoturismo y turismo etnográfico.

Enoturismo, Oleoturismo, Agroturismo y turismos específicos ligados a los productos agroalimentarios de calidad certificadas de la provincia. Turismo gastronómico.

Turismo rural vivencial y experiencial. 


\begin{tabular}{|l|}
\hline Turismo de embalsesy zonas húmedas. \\
\hline Herencia cultural y turismo religioso. Turismo Halal y Turismo Kosher. \\
\hline Turismo responsable y comunitario. \\
\hline
\end{tabular}

Elaboración propia

\section{Observatorio de Turismo Gastronómico de Córdoba}

El Observatorio de Turismo Gastronómico es un proyecto específico del CAPT que tiene como objeto hacer accesible los conocimientos turístico-gastronómicos a los diversos agentes implicados en el fenómeno de la gastronomía que les permita:

- Una toma de conciencia de la riqueza de los saberes y capacidades de que es depositaria la gastronomía cordobesa como factor clave del turismo cultural

- El aprovechamiento compartido del conocimiento gastronómico en sus dimensiones económicas, culturales y societales.

- La disponibilidad del conocimiento gastronómico como bien público y la visibilización, difusión y reconocimiento de la diferenciación y calidad gastronómica de Córdoba y provincia.

Por tanto, el observatorio de turismo gastronómico pretende ser útil para la diversidad de agentes sociales involucrados en el fenómeno de la gastronomía, a través de esta línea de investigación que aporte conocimiento a la ciudadanía y al sector empresarial con objeto de que le sirva de base para la innovación y aplicación del conocimiento y a las asociaciones gastronómicas que puedan promover cambios, iniciativas o trasformaciones en beneficio social.

\section{Difusión de resultados}

Los resultados del trabajo de investigación de los miembros del CAPT serán ampliamente difundidos y publicados en la página web (http://www.uco.es/sitcor/) diseñada como instrumento de transferencia del conocimiento para investigadores, profesores, técnicos, gestores políticos y empresarios y población en general, así como un recopilatorio de informes, estudios y balances de elaboración propia y de otras entidades. Asimismo, se petenden divulgar sus resultados a través de redes sociales en Internet, grupos y redes de investigación y conocimiento en turismo, Universidades y medios de comunicación profesionales de turismo, asociaciones empresariales del sector, etc.

\section{Imagen 1. Web CAPT-SITCOR}

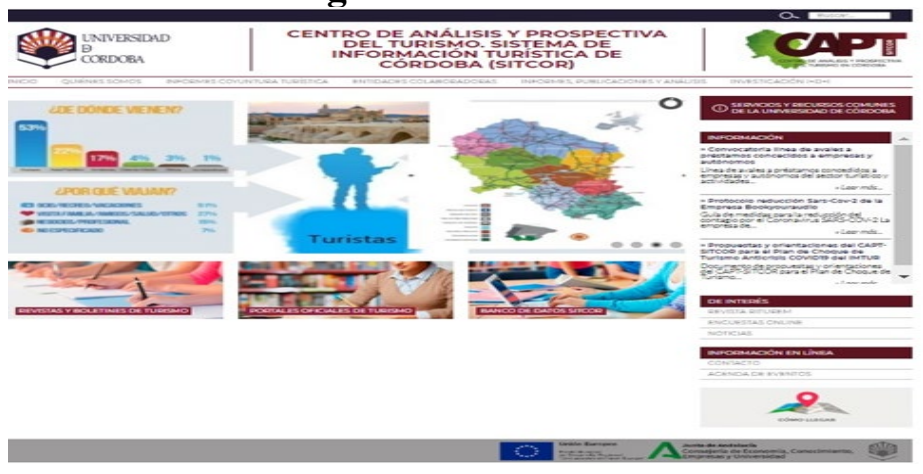

Fuente: Portal web oficial del CAPT. 
En el primer semestre de este año, se han publicado dos documentos de elaboración propia: una primera nota informativa con el balance del sector turístico en la provincia para el primer trimestre del presente año 2020 y un documento de propuestas y orientaciones del CAPT-SITCOR para el Plan de choque de turismo anticrisis Covid-19 del Instituto Municipal de Turismo del Ayuntamiento de Córdoba.

\section{Conclusión}

En definitiva, la puesta en marcha del Centro de Análisis y Prospectiva del Turismo (CAPT) de la Universidad de Córdoba supone la creación de un instrumento de asesoramiento y transferencia del conocimiento para al sector empresarial y profesional y para las Administraciones Públicas con competencia en materia turística que creemos que puede contribuir de manera a la sostenibilidad y competitividad del sector turístico cordobés en particular y del andaluz en general. Y con ello puede aportar, sin duda, una radiografía continua de la situación, coyuntura y pulso del turismo en Córdoba no meramente como un observatorio turístico tradicional, sino con una orientación más integral y aplicada hacia la puesta en marcha de estrategias de planificación, gobernanza, promoción y comercialización, formación, investigación, desarrollo e innovación para un destino turístico responsable y sostenible partiendo de las experiencias contrastadas y el conocimiento obtenido de los Grupos de Investigación y expertos de la Universidad de Córdoba. 